\title{
The Network for Intracortical Communication in Mouse Visual Cortex
}

\author{
Andreas Burkhalter
}

\begin{abstract}
New techniques for identifying cell types, tracing their synaptic partners, imaging and manipulating their activity in behaving organisms have made mice a widely used model for linking brain circuits to behavior. Most behaviors are tied to vision: identifying objects, guiding movements of body parts, navigating through the environment, and even social interactions. Reason enough to focus on the mouse visual cortex. To find our way around in the occipital cortex, we needed a map. We took a classic approach and traced in the same animal the outputs from multiple retinotopic sites of primary visual cortex (V1) and compared the relative location of projections in the extrastriate cortex. We found nine extrastriate maps and showed by single unit recordings that each of the connectional maps contained visually responsive neurons whose receptive fields were mapped in orderly fashion and completely covered the visual field. Remarkably, a tiny region of one sixth of a dime contained a two- to three-times larger number of areas than the highly developed somatosensory and auditory cortices. By tracing the connections, we found that each of the ten visual areas projected to 25-35 cortical targets and interconnected virtually all of the areas reciprocally with one another. Although the binary graph density of the connection matrix was nearly complete, the connection strengths between areas within the ventral and dorsal cortex differed, indicating that the information from V1 flowed into distinct but interconnected streams. Unit recordings and calcium imaging studies showed that the ventral and dorsal streams processed different spatiotemporal information, which aligned with known properties of streams in primates. Analyses of the laminar patterns of interareal projections showed that areas were organized at multiple levels, suggesting that each stream represented a processing hierarchy.
\end{abstract}

\footnotetext{
A. Burkhalter $(\bowtie)$

Department of Neuroscience, Washington University School of Medicine, St. Louis, MO 63110, USA

e-mail: burkhala@pcg.wustl.edu 


\section{Introduction}

Over the past decades, neuroscience research has shown that sensory inputs are processed at multiple locations distributed across the brain. These regions do not encode specific mental faculties but are responsible for specific unitary operations (Kandel and Hudspeth 2013). Cognition arises in a network of serial and parallel pathways between functionally discrete units, each responsible for elements-but not all aspects - of a given function. Although the tenet of functional localization holds that neural processing is modular, the structure of the underlying network and the rules of interareal communication are not well understood. Thanks to the development of powerful new tools for recording, labeling and genetically manipulating brain circuits, the mouse visual system has emerged as a tractable system in which these questions can be addressed with unprecedented precision (Luo et al. 2008; Huberman and Niell 2011; Oh et al. 2014; Zingg et al. 2014).

Mice are most active at night and rely heavily on their whiskers for recognizing objects and their ears and noses for hearing and social communication (Holy and Guo 2005; Jadhav and Feldman 2010; Stowers et al. 2013). When starved for food, mice are diurnal and use dichromatic vision for guiding their actions in the field (Jacobs et al. 2004; Daan et al. 2011; Baden et al. 2013). Through their small eyes with afoveate retinas, the world looks blurred and lacks the rich detail experienced by humans, whose vision is 100 times sharper. At close range, however, the acuity of 0.5 cycles/deg is sufficient to resolve landmark features that can be used for referencing locomotion-dependent path integration signals during spatial navigation (Prusky et al. 2000; Prusky and Douglas 2005; Chen et al. 2013). In fact, experiments on visual object recognition have shown that rats, and presumably mice, can use invariant shape information to identify landmarks from a variety of different viewing angles (Alemi-Neissi et al. 2013). These studies demonstrate that mice process multiple complex visual cues and associate them with motor actions. Many of these computations are performed in interconnected cortical and subcortical networks, bringing up the questions of what the architecture of these networks is and how do functionally distinct areas communicate with each other.

\section{Thalamocortical Projections to Mouse Visual Cortex}

The visual cortex receives thalamic input from the lateral geniculate (LGN) and the lateral posterior (LP) nuclei. LGN inputs to V1 terminate most densely in layers 3 and 4, and more sparsely in layer 1 and at the layer 5/6 border (Dräger 1974; Antonini et al. 1999). In addition, sparse projections from the LGN terminate in the lateral extrastriate areas but avoid medial extrastriate cortex (Antonini et al. 1999). V1 also receives thalamocortical inputs from LP, which terminate in layers 1 and 5. LP inputs to surrounding extrastriate cortex terminate in layers 1, 3, 4 and 6 (Hughes 1977; Herkenham 1980). Although the extrastriate target areas of 
these connections were not positively identified, the results show that thalamocortical inputs from thalamic relays are deployed to V1 as well as to surrounding extrastriate cortex (Sanderson et al. 1991). With this thalamocortical input in place, it is not surprising that expression of the activity-dependent immediate early gene, Arc, shows that much of the thalamorecipient cortex is driven by visual input (Burkhalter et al. 2013).

\section{Cortical Cartography}

Inspired by the emerging field of genetics of the mouse brain (Sidman et al. 1965), Caviness (1975) rang in the modern era of mouse cartography. Refining the surfacebased maps of lissencepahlic mouse cortex constructed by the classic 'cytoarchitects' (Woolsey 1967), Caviness introduced the flatmap format that displayed the cortex in a single map that preserved the natural topology of parcels (Van Essen 2013). In this map, 26 neocortical parcels were identified and, for the first time, clearly showed the shape and extent of V1, including the surrounding extrastriate areas 18a and 18b. A more detailed surface-based map based on the Allen Reference Atlas identified 34 cytoarchitectonic parcels (Dong 2008; Ng et al. 2010), which is similar to the 37 parcels identified in a widely used slicebased atlas by Franklin and Paxinos (2007). Using a variety of histochemical and immunological markers in tangential sections of physically flattened cortex, we were able to identify only 23 parcels, but many with much greater confidence than possible with the classic Nissl stain (Wang et al. 2011, 2012). Notably, our parcellation scheme falls short in the auditory, posterior parietal and visual cortices, which are at the very locations in which we found multiple topographic maps (Wang and Burkhalter 2007). Thus, it appears that some of the areas annotated in the atlases are inspired by our area map, but in reality their borders are too subtle to be identified with confidence by cytoarchitectonic criteria.

\section{Areal Organization of Visual Cortex}

Early topographic mapping studies using microelectrodes showed that extrastriate cortex surrounding V1 contains multiple orderly maps of the visual field (Dräger 1975; Wagor et al. 1980). The conclusion from the layout of the visuotopic maps was that V1 is adjoined on the lateral side by area V2, which is flanked by V3. On the medial side, V1 is adjoined by two additional maps, a rostral area Vm-r and a caudal area Vm-c (Wagor et al. 1980). This primate-inspired areal layout was soon challenged by the discovery that V1 projection targets vastly outnumbered the reported visuotopic areas (Olavarria and Montero 1989). In the eyes of some investigators, the mismatch argued against an organization in which V1 was surrounded by a string of areas and favored a scheme in which the projection 
patches represented inputs to distinct modules within a single area (Kaas et al. 1989). With rodents rapidly taking center stage in neuroscience, the time was ripe to revisit the issue. By labeling the connections of two to three distinct visuotopic locations of V1 with different tracers in the same animal, making sideby-side comparisons of projections in extrastriate cortex and mapping receptive fields, we produced maps of rat and mouse visual cortex (Coogan and Burkhalter 1993; Wang and Burkhalter 2007; Fig. 1). In both species we found maps that strongly argued against the primate-inspired scheme proposed by Wagor et al. (1980), in which a single large area surrounded lateral and rostral V1. Instead,

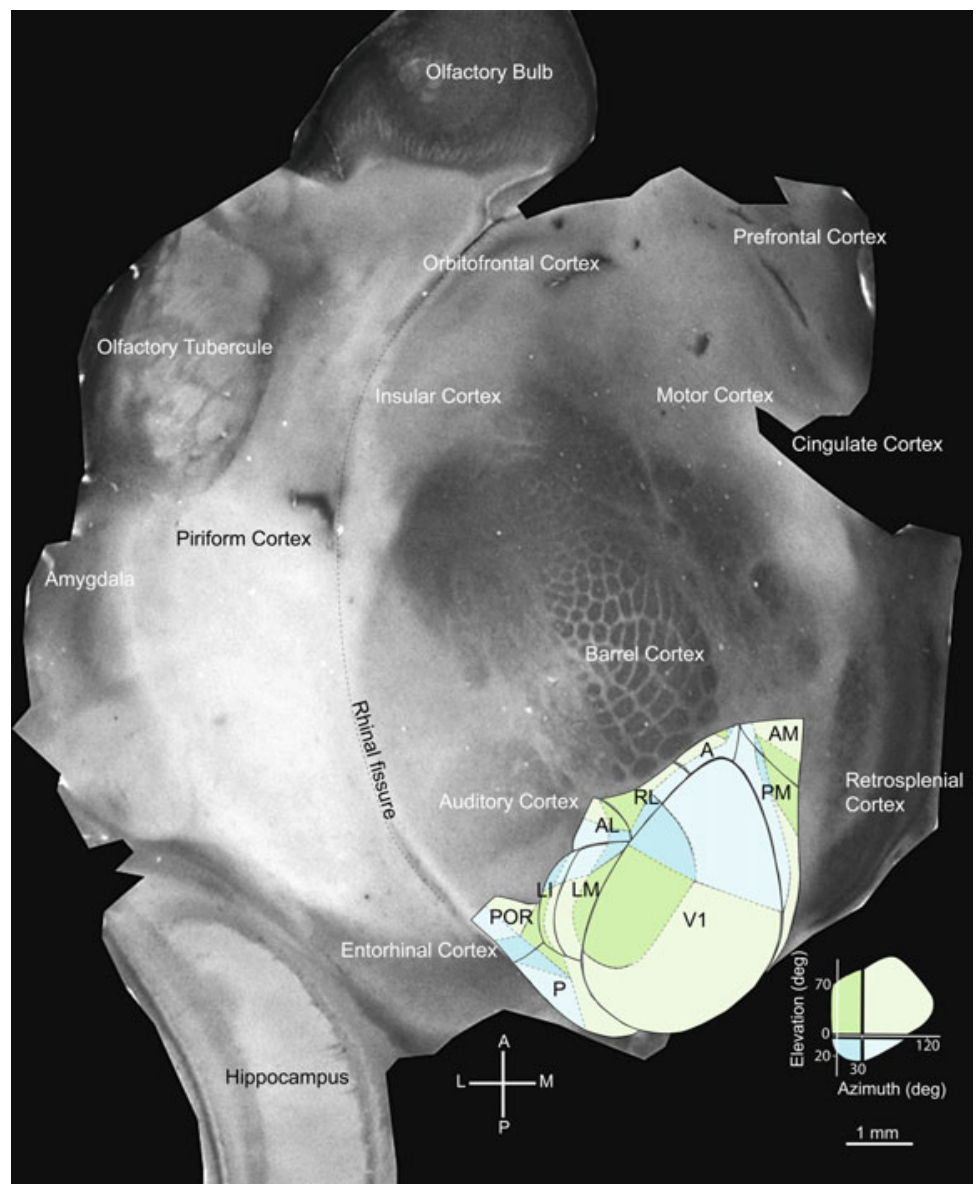

Fig. 1 Area map of mouse visual cortex. Tangential section through flatmounted left cortical hemisphere stained with an antibody against the muscarinic type 2 acetylcholine receptor. The different colors indicate different quadrants of the right visual field. Abbreviations: $A$ anterior area, $A L$ anterolateral area, $A M$ anteromedial area, $L I$ laterointermediate area, $L M$ lateromedial area, $V I$ primary visual cortex, $P$ posterior area, $P M$ posteromedial area, $P O R$ postrhinal area, $R L$ rostrolateral area, $A$ Anterior, $L$ lateral, $M$ medial, $P$ posterior 
the results showed an organization in which V1 was surrounded by a string of small areas that each contained a complete map of the visual field. This finding suggested that ancestral cortex had a complex organization and that select areas identified in primates might be homologous to primordial extrastriate areas in rodents (Rosa and Krubitzer 1999). One of these may be the lateromedial area (LM), which is the only area that shares the vertical meridian with V1 and, for that reason, resembles V2 in primates (Allman and Kaas 1971). But, unlike V2, which has a split horizontal meridian representation, the map in LM is topologically equivalent to the visual field. In fact, this is true for every visuotopic map we have identified, which all show that the margins of the visual field are mapped along the areal borders. To minimize the length of the connections between areas, matching topographic locations in different maps are aligned across shared borders. One of the lessons from these studies is that extrastriate cortex surrounding V1 contains a larger number of areas than annotated in widely used atlases (Franklin and Paxinos 2007; Dong 2008) and employed as references for the mesoscale connectome (Oh et al. 2014; Zingg et al. 2014). It is important to note that, except for the V1 border, which is readily detected in Nissl-stained sections, the cytoarchitecture of the surrounding extrastriate cortex is remarkably uniform. The single exception is the LM/anterolateral area (AL) border, which can be identified by Nissl staining but only when the eyes are keyed to the cytoarchitectonic transition, highlighted by the expression of the muscarinic type 2 acetylcholine receptor (Wang et al. 2011). However, perhaps the most surprising result is that the mouse visual cortex, which is one third the size of barrel cortex, contains at least ten areas, seven more than the somatosensory cortex. One interpretation of the unexpected multitude of visuotopic maps is that vision for perception and for guiding motor actions arises from a larger number of unitary operations than somatosensation and that these elementary processes are represented in different visual areas.

\section{Interareal Connections}

To study the interareal network of visual cortex, we injected the anterograde tracer biotinylated dextran amine (BDA) into ten areas, which we identified by their location relative to callosal landmarks (Wang et al. 2012). Projections to 40 targets were identified based on a combination of cytoarchitectonics and the expression of various molecular markers. The projection strength of each pathway was determined by the optical density of labeled axon branches and terminals in the target zone relative to the total output. Earlier studies have shown that optical density is tightly correlated with bouton density (Wang et al. 2011). The results of the $10 \times 40$ connectivity matrix show 307 of 390 possible linkages (79\%), which accounts for a $13 \%$ higher graph density than in the macaque cortex (Markov et al. 2013). The connection density within the visual cortex proper is even higher, showing that virtually all of the ten visuotopically organized areas are interconnected reciprocally with one another (Wang et al. 2012). The connection strengths span at least 
three orders of magnitude, showing a long-tailed distribution with small numbers of strong and a large numbers of weak connections. Although the connection strength in mouse cortex varies over a narrower range than in macaque (Markov et al. 2011), the lognormal distribution found in both species indicates that the fundamental principles of cortical connectivity are evolutionarily conserved.

In primates, visual information is processed in dorsal and ventral cortical streams specialized for 'where' an object is located or for guiding actions and 'what' an object is (Ungerleider and Mishkin 1982; Goodale and Milner 1992). If such streams exist in mice, how do they arise from a network with seemingly low binary specificity? One way this might be achieved is by routing the flow of information through pathways with different connection strengths. Consistent with this notion, we found that each source area of visual cortex had a unique profile of connection strengths. We assessed between-area similarities and found that the projection strengths among dorsal and ventral networks were distinct. The dorsal network consisted of areas $\mathrm{AL}$, rostrolateral area $(\mathrm{RL})$, anteromedial area (AM), posteromedial area (PM) and anterior area (A), whereas V1, LM, laterointermediate area (LI), postrhinal area (POR) and posterior area $(\mathrm{P})$ were grouped in the ventral network (Wang et al. 2012). Although streams were revealed in the graph of cortex-wide connections, we wondered whether they were present in the $10 \times 10$ connectivity graph of visuotopically organized areas. The graph of projection strengths clearly grouped areas into dorsal (i.e., AL, RL, AM, PM, A) and ventral (i.e., V1, LM, LI, POR, P) communities in which connections within modules were twice as strong as those between modules. Within modules, the shortest pathways were always direct. By contrast, the shortest pathways between modules were often indirect, which means that the combined strength of the indirect path was stronger that the direct path. Thus, for communication between modules, the most effective path may be indirect. Interestingly, not a single short path linking the two modules travels through V1, indicating that, similar to cat and monkey (Sporns et al. 2007), V1 is not a network hub for interareal communication. Instead, judged by the number of connections, this role belongs to area LM. Although lower in the hierarchy than monkey V4, which has a similar status in the network, LM may play a critical role in integrative processing of visual information.

\section{Cortical Hierarchy}

The idea that hierarchical relationships between areas of mouse visual cortex can be derived from the laminar organization of connections goes back to analyses of primate cerebral cortex (Felleman and Van Essen 1991; Markov et al. 2014). In monkey, it was noticed that many reciprocal connections consisted of feedforward projections terminating in layer 4 and feedback projections terminating outside of layer 4. Such asymmetrical linkages are present between most reciprocally connected pairs of cortical areas. In rat and mouse, reciprocal interareal connections share many of the features found in primate (Coogan and Burkhalter 1990, 1993; 
Dong et al. 2004a). However, unlike in primates, feedforward axonal projections from V1 are not restricted to layer 4. Instead, the projections terminate in a column across all layers. Importantly, however, feedforward connections always include layer 4. In contrast, feedback projections from surrounding extrastriate cortex to layer 4 of V1 are extremely sparse and preferentially terminate in layers 1, 2, 3, 5 and 6. Thus, the asymmetry in the innervation strength of layer 4 is the hallmark feature of reciprocal interareal connections. While these are striking similarities to feedforward and feedback connections in monkey, it is important to note that the columnar pattern of feedforward connections in rodents differs from that in monkey, which is restricted to layer 4. In fact, rodent feedforward connections resemble more closely the lateral connections in monkey (Felleman and Van Essen 1991). A likely reason for this difference is that feedforward connections in rodents originate from layers 2-6 (Coogan and Burkhalter 1988), whereas, in monkey, layers 2 and 3 are the main sources of these connections (Felleman and Van Essen 1991). From a developmental perspective, Dehay and Kennedy (2007) have argued that layers 2 and 3 in primates are different from layers 2 and 3 in mice, which lack the computational components of primate cortex. Abandoning input from deep layers in feedforward connections and increasingly relying on inputs from layers 2 and 3 may be a structural manifestation of the superior sophistication in interareal communication in primates.

In primates, different hierarchical levels are associated with different stages of visual processing (Felleman and Van Essen 1991). One way stimulus complexity is expressed is by the convergence of input reflected in the size of receptive fields. Recordings in mouse visual cortex show that receptive fields in V1 are small (10 deg) and increase across different extrastriate visual areas to reach a size that covers most of the visual field (Wang and Burkhalter 2007). Indirect support for an areal hierarchy also comes from the pattern of subcortical connections. For example, only areas $\mathrm{V} 1$ and LM receive input from the main afferent LGN nucleus (Oh et al. 2014). Thalamocortical inputs to all other visual areas originate from the LP nucleus (Oh et al. 2014). In addition, projections from V1 to the superior colliculus terminate in the most superficial sensory layers, whereas the outputs from higher areas are sent to deeper visuomotor layers (Coogan and Burkhalter 1993; Wang and Burkhalter 2013).

\section{Synaptic Organization of Feedforward and Feedback Connections}

Signatures for a cortical hierarchy are also observed in the distinct synaptic connectivity of feedforward and feedback connections. Both types of interareal connections are made by excitatory, glutamatergic pyramidal cells (Johnson and Burkhalter 1994; Domenici et al. 1995; Dong et al. 2004b), whereas long-range projections of GABAergic neurons are negligible (McDonald and Burkhalter 1993; 
but see Caputi et al. 2013). In rat and mouse, feedforward and feedback connections to higher (i.e., LM) and lower visual areas (i.e., V1) provide monosynaptic input to pyramidal cells and GABAergic neurons (Dong et al. 2004b). Among the targets in layers 2 and 3, we found a handful of somatostatin- and calretinin-expressing interneurons but the vast majority of GABAergic cells expressed parvalbumin (Gonchar and Burkhalter 1999, 2003). Thus, in the target region, responses of pyramidal cells to excitatory feedforward and feedback inputs are influenced by disynaptic feedforward inhibition from parvalbumin neurons. Although the laminar projection pattern of feedforward and feedback circuits are distinct (Dong et al. 2004a), structurally the circuits for feedforward inhibition are similar in both pathways (Gonchar and Burkhalter 1999). Physiologically, however, the responses of pyramidal cells to feedforward inputs are opposed by stronger inhibition than the responses to feedback inputs (Shao and Burkhalter 1996; Dong et al. 2004b). The reasons for the pathway-specific excitatory/inhibitory balance are that feedforward inputs to parvalbumin-expressing neurons are relatively stronger than to pyramidal cells whereas feedback inputs to both types of cells are similar (Yang et al. 2013). The stronger excitation of parvalbumin neurons is probably due to signaling via calcium-permeable GluR2-lacking AMPA receptors that elicit large quantal amplitude responses with fast kinetics (Hull et al. 2009). By contrast, feedforward inputs to pyramidal cells are mediated by slow, smallamplitude AMPA receptors (Hull et al. 2009). The result of the fast/large amplitude AMPA-mediated currents at feedforward inputs onto parvalbumin-expressing neurons is that feedforward inhibition is initiated reliably and in a precisely timed manner. In contrast, small amplitude and slower AMPA-mediated currents at feedback synapses facilitate integration of convergent inputs onto pyramidal neurons. The motif of feedforward inhibition not only balances excitation but influences circuit gain and dynamics (Kepecs and Fishell 2014). We can only speculate what effects diverse feedforward inhibition might have on the processing of visual signals in feedforward and feedback circuits. In the static mode, feedforward circuits are good for selecting correlated inputs. Computational modelling has shown that this enhances stimulus detection and improves the accuracy of stimulus representation, whereas, in the default mode, the feedback circuit may improve response probability to sensory input (Kremkow et al. 2010). However, when top-down attention is focused on a stimulus, the excitatory/inhibitory balance may change and improve the accuracy of stimulus detection (Wang et al. 2013).

\section{Dorsal and Ventral Processing Streams}

Motivated by the perplexing number of visual areas and their striking connectivity within hierarchically organized dorsal and ventral streams, it was natural to search for analogies to the distributed processing within 'where' and 'what/action' streams of primates (Ungerleider and Mishkin 1982; Goodale and Milner 1992). The proposal that rat cortex contains distinct streams that are specialized for visual 
guidance and object recognition was made almost 25 years ago (Kolb 1990). Since then, numerous studies have shown that deficits in pattern discrimination were associated with lesions in lateral extrastriate visual cortex, whereas damage to cortex anterior and medial to $\mathrm{V} 1$ affected polysensory integration and spatial navigation (Kolb and Walkey 1987; Wong and Brown 2006; Torrealba and Valdes 2008; Zhang et al. 2010). But the lesioning techniques used in these studies did not afford the spatial resolution for linking the behavioral deficit unequivocally to specific areas, a problem that will likely be overcome by optogenetic approaches (Lien and Scanziani 2013). Recently, significant progress was made by two-photon imaging of calcium transients in upper layer neurons of multiple areas in mouse visual cortex (Andermann et al. 2011; Marshel et al. 2011; Roth et al. 2012). These recordings showed that tuning to high spatial frequency was more common in LI than in AL, RL and AM, which are more selective for high temporal frequency and the direction of motion. Although these findings are broadly consistent with the concept that ventral stream areas are specialized for image detail and dorsal stream areas preferentially respond to transient inputs (Van Essen and Gallant 1994), the results show inconsistencies. For example, neurons of the ventral stream area, LM, have low spatial acuity and are tuned to high temporal frequencies. It is possible that, similar to V2 of primates (Nassi and Callaway 2009), LM consists of functionally distinct compartments and the true response properties were masked by averaging across modules. Further, counter to the prediction, neurons in the dorsal stream area, PM, have high spatial acuity and prefer longer-lasting, slow moving objects. One way to explain these inconsistencies is that high spatial acuity and sensitivity to slow visual motion recorded in PM provides landmark information, which is used to calibrate distance and direction signals from locomotion used for path integration (Harvey et al. 2012; Saleem et al. 2013).

Although distinct streams are observed in the cortex, functionally distinct channels emerge from the retina, are present in the LGN and can be traced throughout the afferent visual pathway to V1 (Piscopo et al. 2013; Cruz-Matin et al. 2014; Dhande and Huberman 2014). In V1, neural responses represent a weighted combination of inputs from parallel afferent geniculocortical pathways and feedback inputs from higher cortical areas with distinct spatiotemporal properties (Gao et al. 2010). From V1, impulses are sent to different areal streams. The question then is whether the functional differences arise in V1 or are generated in the dorsal or ventral areas to which V1 sends its output. To address this question, Glickfeld et al. (2013) labeled striate cortical inputs from V1 with the calcium indicator GCaMP3.3 and imaged calcium transients in axon terminals projecting to LM, AL and PM. The results show that the visual preferences of each projection are different and matched those of neurons in the target area, suggesting that each area inherits the response properties from functionally specialized neurons in V1. The important conclusion of this work is that different V1 neurons transmit information tailored to its projection target. This organization is consistent with our observation that individual V1 neurons largely lack collateral projections and project to single area of extrastriate cortex (Wang and Burkhalter 2005). More recently, similar results have been reported in the connectivity between V1, LM and AL, supporting 
the idea that interareal transmission relies on dedicated neuronal connections (Berezovskii et al. 2011). The overall conclusion of these studies is that the binary specificity of the network of interareal connection might be much greater than indicated by pathway tracing of connections with tracers that lack cellular specificity.

Acknowledgments This work was supported by National Eye Institute Grants RO1EY-05935, RO1EY-016184, RO12EY20525 and RO1EY22090, the McDonnell Center for Systems Neuroscience and the Human Frontier Science Program. We thank Greg De Angelis, Yarimar Carrasquillo, Luciano Domenici, Hongwei Dong, Enquan Gao, Yuri Gonchar, Bryan Hooks, Weiqing Ji, Jeanne Nerbonne, Zhengwei Shao, Katia Valkova, Quanxin Wang, Akiko Yamashita and Weiguo Yang for their contributions.

Open Access This chapter is distributed under the terms of the Creative Commons AttributionNoncommercial 2.5 License (http://creativecommons.org/licenses/by-nc/2.5/) which permits any noncommercial use, distribution, and reproduction in any medium, provided the original author(s) and source are credited.

The images or other third party material in this chapter are included in the work's Creative Commons license, unless indicated otherwise in the credit line; if such material is not included in the work's Creative Commons license and the respective action is not permitted by statutory regulation, users will need to obtain permission from the license holder to duplicate, adapt or reproduce the material.

\section{References}

Alemi-Neissi A, Roselli FB, Zoccolan D (2013) Multifeatural shape processing in rats engaged in invariant object recognition. J Neurosci 33:5939-5956

Allman JM, Kaas JH (1971) Representation of the visual field in striate and adjoining cortex of the owl monkey (Aotus trivirgatus). Brain Res 35:89-106

Andermann ML, Kerlin AM, Roumis DK, Glickfeld LL, Reid RC (2011) Functional specialization of mouse higher visual cortical areas. Neuron 72:1025-1039

Antonini A, Fagolini M, Stryker MP (1999) Anatomical correlates of functional plasticity in mouse visual cortex. J Neurosci 19:4388-4408

Baden T, Schibert T, Chang L, Wei T, Zaichuk M, Wissinger B, Euler T (2013) A tale of two domains: near-optimal sampling of achromatic contrasts in natural scenes through asymmetric photoreceptor distribution. Neuron 80:1206-2017

Berezovskii VK, Nassi JJ, Born RT (2011) Segregation of feedforward and feedback projections in mouse visual cortex. J Comp Neurol 519:3672-3683

Burkhalter A, Sporns O, Gao E, Wang Q (2013) Network of mouse visual cortex. In: Werner JS, Chalupa LM (eds) The new visual neurosciences. MIT Press, Cambridge, MA, pp 243-254

Caputi A, Melzer S, Michael M, Monyer H (2013) The long and short of GABAergic neurons. Curr Opin Neurobiol 23:1-8

Caviness VS (1975) Architectonic map of neocortex of the normal mouse. J Comp Neurol 164:247-263

Chen G, King JA, Burgess N, O'Keefe J (2013) How vision and movement combine in the hippocampal place code. Proc Natl Acad Sci USA 110:378-383

Coogan TA, Burkhalter A (1988) Sequential development of connections between striate and extrastriate visual cortical areas in the rat. J Comp Neurol 278:242-252

Coogan TA, Burkhalter A (1990) Conserved patterns of cortico-cortical connections define areal hierarchy in rat visual cortex. Exp Brain Res 80:49-53 
Coogan TA, Burkhalter A (1993) Hierarchical organization of areas in rat visual cortex. J Neurosci 13:3749-3772

Cruz-Matin A, El-Danaf RN, Osakada F, Sriram B, Dhande OS, Ngyuen PL, Callaway EM, Gosh A, Huberman AD (2014) A dedicated circuit links direction-selective retinal ganglion cells to the primary visual cortex. Nature 507:358-361

Daan S, Spoelstra K, Albrecht U, Schmutz I, Daan M, Daan B, Rienks F, Poletaeva 1, Dell'Omo G, Vyssotski A, Lipp HP (2011) Lab mice in the field: unorthodox daily activity and effects of dysfunctional circadian clock allele. J Biol Rhythms 26:118-129

Dehay C, Kennedy H (2007) Cell-cycle control and cortical development. Nat Rev Neurosci 8:438-450

Dhande OS, Huberman AD (2014) Retinal cell maps in the brain: implications for visual processing. Curr Opin Neurobiol 24:133-142

Domenici L, Harding GW, Burkhalter A (1995) Patterns of synaptic activity in forward and feedback pathways within rat visual cortex. J Neurophysiol 74:2649-2664

Dong HW (2008) Allen reference atlas. A digital color atlas of the C57BL/6 J male mouse. Wiley, Hoboken, NJ

Dong H, Wang Q, Valkova K, Gonchar Y, Burkhalter A (2004a) Experience-dependent development pf feedforward and feedback circuits between lower and higher areas of mouse visual cortex. Vis Res 44:3389-3400

Dong H, Shao Z, Nerbonne JM, Burkhalter A (2004b) Differential depression of inhibitory synaptic responses in feedforward and feedback circuits between different areas of muse visual cortex. J Comp Neurol 475:361-373

Dräger UC (1974) Autoradiography of tritiated proline and fucose transported transneuronally from the eye to the visual cortex in pigmented and albino mice. Brain Res 82:284-292

Dräger UC (1975) Receptive field of single cells and topography in mouse visual cortex. J Comp Neurol 160:269-287

Felleman DJ, Van Essen DC (1991) Distributed hierarchical processing in the primate cerebral cortex. Cereb Cortex 1:1-47

Franklin KBJ, Paxinos G (2007) The mouse brain in stereotaxic coordinates. Elsevier, Amsterdam

Gao E, De Angelis GC, Burkhalter A (2010) Parallel input channels to mouse primary visual cortex. J Neurosci 30:5812-5926

Glickfeld LL, Andermann ML, Bonin V, Reid RC (2013) Cortico-cortical projections in mouse visual cortex are functionally target specific. Nat Neurosci 16:219-226

Gonchar Y, Burkhalter A (1999) Differential subcellular localization of forward and feedback interareal inputs to parvalbumin expressing GABAergic neurons in rat visual cortex. J Comp Neurol 406:346-360

Gonchar Y, Burkhalter A (2003) Distinct GABAergic targets of feedforward and feedback connections between lower and higher areas of rat visual cortex. J Neurosci 23:10904-10912

Goodale MA, Milner AD (1992) Separate visual pathways for perception and action. Trends Neurosci 15:20-25

Harvey CD, Coen P, Tank DW (2012) Choice-specific sequences in parietal cortex during a virtual-navigation decision task. Nature 484:62-68

Herkenham M (1980) Laminar organization of thalamic projection to the rat neocortex. Science 207:532-535

Holy TE, Guo Z (2005) Ultrasonic songs of male mice. PLoS Biol 3(12):e386

Huberman AD, Niell CM (2011) What can mice tell us about how vision works? Trends Neurosci 34:464-473

Hughes HC (1977) Anatomical and neurobehavioral investigations concerning the thalamocortical organization of the rat's visual system. J Comp Neurol 175:311-335

Hull C, Isaacson JS, Scanziani M (2009) Postsynaptic mechanisms govern the differential excitation of cortical neurons by thalamic inputs. J Neurosci 29:9127-9136

Jacobs GH, Williams GA, Fenwick JA (2004) Influence of cone pigment coexpression on spectral sensitivity and color vision in the mouse. Vis Res 44:1615-1622

Jadhav SP, Feldman DE (2010) Texture coding in the whisker system. Curr Opin Neurobiol 20:313-318 
Johnson R, Burkhalter A (1994) Evidence for excitatory amino acid neurotransmitters in forward and feedback corticocortical pathways within rat visual cortex. Eur J Neurosci 6:272-286

Kaas JH, Krubitzer LA, Johanson KL (1989) Cortical connections of areas 17 (V-I) and 18 (V-II) of squirrels. J Comp Neurol 428:337-354

Kandel ER, Hudspeth AJ (2013) The brain and behavior. In: Kandel ER, Schwartz JH, Jessel TM, Siegelbaum SA, Hudspeth AJ (eds) Principles of neural science, 5th edn. McGraw Hill, New York, NY, pp 5-18

Kepecs A, Fishell G (2014) Interneuron cell types are fit to function. Nature 505:318326

Kolb B (1990) Posterior parietal and temporal association cortex. In: Kolb B, Tees RC (eds) The cerebral cortex of the rat. MIT Press, Cambridge, MA, pp 459-471

Kolb B, Walkey J (1987) Behavioral and anatomical studies of the posterior parietal cortex in the rat. Behav Brain Res 23:127-145

Kremkow J, Perrinet LU, Masson GS, Aertsen A (2010) Functional consequences of correlated excitatory and inhibitory conductances in cortical networks. J Comput Neurosci 28:579-594

Lien AD, Scanziani M (2013) Tuned thalamic excitation is amplified by visual cortical circuits. Nat Neurosci 16:1315-1323

Luo L, Callaway EM, Svoboda K (2008) Genetic dissection of neural circuits. Neuron 57:634-660

Markov NT, Misery P, Falchier A, Lamy C, Vezli J, Quilodran R, Gariel MA, Giroud P, ErcseyRavaz M, Pilaz LJ, Huissoud C, Barone P, Dehay C, Toroczkai Z, Van Essen DC, Kennedy H (2011) Weight consistency specifies regularities of macaque cortical networks. Cereb Cortex 21:1254-1274

Markov NT, Ercsey-Ravasz M-M, Lamy C, Gomes ARR, Magrou L, Misery P, Giroud P, Barone P, Dehay C, Toroczkai Z, Knoblauch K, Van Essen DC, Kennedy H (2013) The role of long-range connections on the specificity of the macaque interareal cortical network. Proc Natl Acad Sci USA 110:5187-5192

Markov NT, Vezoli J, Chameau P, Falchier A, Quilodran R, Huissoud C, Lamy C, Misery P, Giroud P, Ullman S, Barone P, Dehay C, Knoblauch K, Kennedy H (2014) Anatomy of hierarchy: feedforward and feedback pathways in macaque visual cortex. J Comp Neurol 522:225-259

Marshel JH, Garret ME, Nauhaus I, Callaway EM(2011) Functional specialization of seven mouse visual cortical areas. Neuron 72:1042-1054

McDonald CT, Burkhalter A (1993) Organization of long-range inhibitory connections within rat visual cortex. J Neurosci 13:768-781

Nassi JJ, Callaway EM (2009) Parallel processing strategies of the primate visual system. Nat Rev Neurosci 10:360-372

Ng L, Lau C, Sunkin SM, Bernard A, Chakrvarty MM, Lein ES, Jones AR, Hawrylycz M (2010) Surface-based mapping of gene expression and probabilistic expression maps in the mouse cortex. Methods 50:55-62

Oh SW, Harris JA, Ng L, Winslow B, Cain N, Mihalas S, Wang Q, Lau C, Kuan L, Henry AM, Mortrud MT, Ouellette B, Nguyen TN, Sorensen SA, Slaughterbeck CR, Wakeman W, Li Y, Feng D, Ho A, Micholas E, Hirokawa KE, Bohn P, Joines KM, Peng H, Hawrylycz MJ, Phillips JW, Hohmann JG, Wohnoutka P, Gerfen CR, Koch C, Bernard A, Dang C, Jones AR, Zeng H (2014) A mesoscale connectome of the mouse brain. Nature 508:207-214

Olavarria J, Montero VM (1989) Organization of visual cortex in the mouse revealed by correlating callosal and striate-extrastriate connections. Vis Neurosci 3:59-69

Piscopo DM, El-Danaf RN, Huberman AD, Niell CM (2013) Diverse visual features encoded in mouse lateral geniculate nucleus. J Neurosci 33:4642-4656

Prusky GT, Douglas RM (2005) Vision. In: Wishaw IQ, Kolb B (eds) The behavior of the laboratory rat. University Press, Oxford, pp 49-59

Prusky GT, West WR, Douglas RM (2000) Behavioral assessment of visual acuity in mice and rats. Vis Res 40:2201-2209

Rosa MGP, Krubitzer LA (1999) The evolution of visual cortex: where is V2? Trends Neurosci 22:242-248

Roth MM, Helmchen F, Kama BM (2012) Distinct functional properties of primary and posteromedial visual area of mouse neocortex. J Neurosci 32:9716-9726 
Saleem AB, Ayaz A, Jeffery KJ, Harris KD, Carandini M (2013) Integration of visual motion and locomotion in mouse visual cortex. Nat Neurosci 16:1864-1869

Sanderson KJ, Dreher B, Gayer N (1991) Prosencephalic connections of striate and extrastriate areas of rat visual cortex. Exp Brain Res 85:324-334

Shao Z, Burkhalter A (1996) Differential balance of excitation and inhibition in forward and feedback circuits of rat visual cortex. J Neurosci 16:7353-7365

Sidman RL, Appel SH, Fuller JF (1965) Neurological mutants of the mouse. Science 150:513-516

Sporns O, Honey CJ, Kötter R (2007) Identification and classification of hubs in brain networks. PLoS One 2:e104910

Stowers L, Cameron P, Keller JA (2013) Ominous odors: olfactory control of instinctive fear and aggression in mice. Curr Opin Neurobiol 23:339-345

Torrealba F, Valdes JL (2008) The parietal association cortex of the rat. Biol Res 41:369-377

Ungerleider LG, Mishkin M (1982) Two cortical systems. In: Ingle DJ, Goodale MA, Mansfield RJW (eds) Analysis of visual behavior. MIT Press, Cambridge, pp 549-586

Van Essen DC (2013) Cartography and connectomes. Neuron 80:775-790

Van Essen DC, Gallant JL (1994) Neural mechanisms of form and motion processing in the primate visual system. Neuron 13:1-10

Wagor E, Mangini NJ, Pearlman AL (1980) Retinotopic organization of striate and extrastriate visual cortex in the mouse. J Comp Neurol 193:187-202

Wang Q, Burkhalter A (2005) Separate output streams from V1 to higher areas of mouse visual cortex. Soc Neurosci Abstr 854:1

Wang Q, Burkhalter A (2007) Area map pf mouse visual cortex. J Comp Neurol 502:339-357

Wang Q, Burkhalter A (2013) Stream-related preferences of inputs to the superior colliculus from area of dorsal and ventral stream of mouse visual cortex. J Neurosci 33:1696-1705

Wang Q, Gao E, Burkhalter A (2011) Gateways of ventral and dorsal streams in mouse visual cortex. J Neurosci 31:1905-1918

Wang Q, Sporns O, Burkhalter A (2012) Network analysis of corticocortical connections reveals ventral and dorsal processing streams in mouse visual cortex. J Neurosci 32:4386-4399

Wang C-T, Lee C-T, Wang X-J, Lo C-C (2013) Top-down modulation on perceptual decision with balanced inhibition through feedforward and feedback inhibitory neurons. PLoS One 8:e62379

Wong AA, Brown RE (2006) Visual detection, pattern discrimination and visual acuity in 14 strains of mice. Genes Brain Behav 5:389-403

Woolsey TA (1967) Somatosensory, auditory and visual cortical areas of the mouse. Johns Hopkins Med J 121:91-112

Yang W, Carrasquillo Y, Hooks BM, Nerbonne JM, Burkhalter A (2013) Distinct balance of excitation and inhibition in an interareal feedforward and feedback circuit of mouse visual cortex. J Neurosci 33:17373-17384

Zhang G-R, Cao H, Kong L, O'Brien J, Baughs A, Jan M, Zhao H, Wang X, Lu X-G, Cook RG, Geller AI (2010) Identified circuit in rat postrhinal cortex encodes essential information for performing specific visual shape discrimination. Proc Natl Acad Sci USA 107:14478-14483

Zingg B, Hintiryan H, Gou L, Song MY, Bay M, Bienkowski MS, Foster NN, Yamashita S, Bowman I, Toga AW, Dong H-W (2014) Neural networks of the mouse neocortex. Cell 156:1096-1111 